\title{
Royal Society warns on hormone disrupters
}

Natasha Loder, London

Britain's science academy is calling for worldwide action to deal with chemicals that may disrupt the body's hormonal functions.

In a report published last week, the Royal Society calls for national and international coordination to deal with the dangers it claims are posed to humans and wildlife by endocrine-disrupting chemicals (EDCs) substances that are thought to mimic or block hormones, in doses too small to trigger a conventional toxic response.

The report, called Endocrine-disrupting Chemicals, was produced by a working group led by the society's vice-president Patrick Bateson, professor of ethology at the University of Cambridge. It says there is strong evidence to link EDC exposure to effects on some organisms. It recommends minimizing human exposure to EDCs - with pregnant women being strongly discouraged from contact with EDCs, such as plasticizers and insecticides.

But research is being hampered by lack of standardization. A US study of 15,000 chemicals, for example, has been unable to begin a planned high-throughput screening programme because researchers have been unable to develop the right assays.

"There are no ways to test these chemicals, no standardized screens or assays, even in one nation," says Theo Colborn, an expert on the endocrine-disrupter hypothesis who works at the World Wildlife Fund in Washington, DC.

Colborn says that new research has recently become available on prenatal exposure to the industrial chemicals polychlorinated biphenyls (PCBs) and co-contaminants, which shows a correlation with reduced IQ, behavioural problems and adverse effects on the immune system.

The British report goes further than one last year from the US National Research Council (NRC), which left an open verdict on the issue of public health risks (see Nature 400,607; 1999).

Some scientists remain sceptical of the endocrine-disrupter hypothesis. Stephen Safe, for example, deputy director of the Center for Environmental and Rural Health at Texas A\&M University and a member of the NRC panel, says that the recent evidence about PCBs is not clear-cut. Safe says that, although he does not necessarily disagree with the endocrine-disrupter hypothesis, he considers that it needs more work, and is not convinced by the evidence so far.

"Do EDCs cause some problems? They have and probably will, but the general hysteria is unwarranted," says Safe. He blames what he calls "synthetic chemical chemophobia" for current public reaction.

In contrast, Bateson says the issue is a "cause for more worry" than genetic modification. He thinks there is a case for making people more aware of the possible dangers, and is arguing for a national body to oversee work on the issue. The society wants to ensure that "sound policies" are developed and warns policy-makers that they must appreciate that the concerns of the public already have some foundation.

The Royal Society report coincides with growing international concern about the harmful effects of EDCs.

In April a meeting of the environment ministers of the G8 group of industrialized countries signed a communiqué stating that the risks posed by hazardous chemical substances were one of the greatest concerns expressed by the people of their countries. The ministers called for a "furtherance of knowledge acquisition on endocrine disrupters through jointly planned and implemented projects and international information sharing".

Colborn is currently touring the world in a bid to raise money to set up an independent international body, which would be funded by industry and government and would coordinate research and expertise.

Earlier this year the UK's Environment Agency published a strategy for reducing potential EDCs. But Bateson thinks European bodies - including those in Britain are "bitty and uncoordinated, with lots of activities all over the place".

\section{Study compares chimps and people}

\section{Robert Triendl, Tokyo}

Scientists at two major Japanese research facilities are finalizing plans for a joint project aimed at comparing the genome of humans with that of chimpanzees.

It will be run by the Brain Science Institute (BSI) and the Genomic Sciences Center (GSC), both of which are part of the Institute of Physical and Chemical Research (RIKEN), Japan's foremost institution for basic science.

The project will be directed by Yoshiyuki Sakaki, who holds joint appointments at the Institute of Medical Science at the University of Tokyo and at the GSC. It will initially concentrate on the comparison of gene clusters expressed in the region within the brain responsible for speech and language.

"It is intellectual ability and especially language that distinguishes humans," says Sakaki. "This project will help us to identify genes that are distinctive of humans and linked to the intellectual capacity of humans."

The project will initially focus on genetic comparison, but a behavioural component may be added. Japan has a rich tradition of ethological research on primates.

Although work on the chimpanzee genome has barely begun, scientists think that experience in sequencing the human genome can be replicated without much problem in the case of chimpanzees.

As structural genomics and research on the mouse genome - areas where Japan has made early commitments - enter the mainstream of genome research, it is hoped that the new project will allow Japanese scientists to pursue an original field of research. "I believe this is an area where Japanese scientists can make a clear difference," says Sakaki.

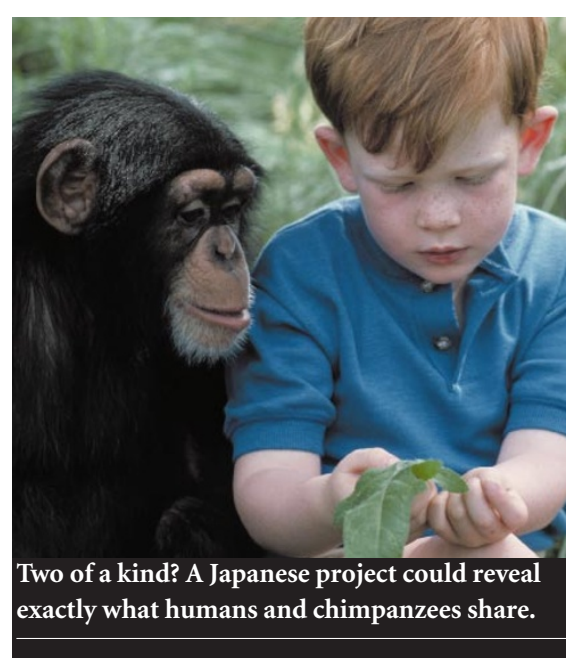

Although funds have not yet been committed, the Science and Technology Agency (STA), which oversees RIKEN, is said to be eager to promote the project for another reason.

At a recent meeting of the international advisory board of the GSC, board members expressed concern that the centre's ample facilities and resources had not been made available to outside scientists.

Both the GSC and the BSI are the result of a shift towards priority-based funding that has gained momentum since the early 1990s. So far, it has not proved easy to integrate the new centres into the diverse landscape of Japanese public research.

According to its director, Masao Ito, the BSI provides small seed funding grants for cooperation with scientists outside the centre. But he acknowledges that these are fairly small, and that it has been difficult to convince officials at the STA to upgrade resources for collaborative research. 